Patricia Whitelock and Russell Cannon, eds.

\title{
The Andromeda Dwarf Spheroidal Galaxies
}

\author{
Taft E. Armandroff \\ Kitt Peak National Observatory, National Optical Astronomy \\ Observatories, ${ }^{1}$ P.O. Box 26732, Tucson, AZ 85726, USA
}

\author{
Gary S. Da Costa \\ Research School of Astronomy \& Astrophysics, Australian National \\ University, Private Bag, Weston Creek P.O., ACT 2611, Australia
}

\begin{abstract}
Our current knowledge of M31's dwarf spheroidal companions is reviewed. Two topics of recent interest constitute the bulk of this review. First, color-magnitude diagrams reaching below the horizontal branch have been constructed for two M31 dwarf spheroidals, based on images from HST/WFPC2. The horizontal branches are predominantly red in both galaxies, redder than expected for their metallicity based on Galactic globular clusters. Thus, the second parameter effect is seen in the M31 halo. Second, recent surveys have revealed three new dwarf spheroidal companions to M31. Thus, dwarf spheroidal galaxies are not as rare around M31 as previously thought and as a result, some properties of the M31 companion system have changed.
\end{abstract}

\section{Introduction}

Dwarf spheroidal galaxies are probably the most common type of galaxy in the Universe. Because of their ubiquity, and because the accretion of dwarf galaxies may have played an important role in the formation/evolution of large galaxies, an understanding of dwarf spheroidal (dSph) galaxies is key to many problems in astronomy. The great majority of our knowledge of dwarf spheroidal galaxies results from the Galaxy's nine dSph companions. In order to broaden this knowledge and to investigate the effects of environment on the formation and evolution of dwarf galaxies, it is important to study dSph galaxies beyond the Milky Way's companions. Some mechanisms by which environment can influence the evolution of dwarf galaxies include: tidal interactions, removal of a dwarf's interstellar medium via ram pressure sweeping, confinement of a dwarf's interstellar medium via ambient pressure, and photoionization of the interstellar medium by a nearby source of UV radiation (such as a quasar or AGN).

Detailed study of dwarf spheroidals beyond the Milky Way will help reveal how much we can generalize from the nine Galactic companions. For example,

\footnotetext{
${ }^{1}$ The National Optical Astronomy Observatories are operated by the Association of Universities for Research in Astronomy under cooperative agreement with the National Science Foundation.
} 
the Galactic dwarf spheroidals follow reasonably tight relations between central surface brightness and absolute magnitude and between absolute magnitude and mean metal abundance (e.g., Caldwell et al. 1992). Are these relations universal or do they change with environment? The present, limited data have not revealed changes with environment (Caldwell et al. 1998). As another example, the Galactic dwarf spheroidals show an amazing diversity in their star formation histories, ranging from a single star-formation episode of globular cluster age to ongoing star formation that peaked $\sim 3$ Gyr ago (e.g., Da Costa 1998). Is this diversity universal or did special circumstances contribute to the varied star formation histories of the Milky Way's dwarf spheroidals? Another puzzle is the (imperfect) correlation among the Galactic dwarfs between the duration of star formation and distance from the Galactic center, with the outer dwarfs continuing to form stars over longer periods than the inner dwarfs (van den Bergh 1994). Again, it would be valuable to understand whether this trend is seen in other systems. Finally, what is the behavior of the galaxy luminosity function at the faint end and is this dependent on environment? There is some indication that rich clusters of galaxies have a steeper slope for the faint end of the luminosity function than the Local Group, though this could be caused by incompleteness in the Local Group (Trentham 1998).

The nearest collection of dwarf spheroidals beyond the Milky Way's halo is M31's companions. Van den Bergh's $(1972,1974)$ survey of 700 square degrees around M31 with the Palomar 48-inch Schmidt and IIIaJ plates revealed three dwarf spheroidal companions (And I, II \& III). The nature of And IV, the fourth object in van den Bergh's discovery list, remains unclear though it is not a dSph companion to M31. It is either a very large open cluster in the outer disk of M31 (e.g. Jones 1993) or a background dwarf galaxy. A nearby bright foreground star makes ground-based observations of this object difficult and we await the outcome of HST WFPC2 imaging being undertaken by A. Ferguson, R. Wyse and J. Gallagher to clarify the nature of this object. Surface brightness profiles and structural parameters have been measured for And I, II \& III by Caldwell et al. (1992). Color-magnitude diagrams from 4m-class ground-based telescopes are also available for all three galaxies (Mould \& Kristian 1990; Konig et al. 1993; Armandroff et al. 1993). These show the brightest $\sim 2$ magnitudes of the red giant branch and yield line-of-sight distances similar to that of M31. All these data indicate a considerable degree of similarity between these three M31 dwarf spheroidals and those of the Galaxy. The most significant developments that have occurred since the last review article devoted exclusively to the M31 dwarf spheroidals (Armandroff 1994) are: 1) color-magnitude diagrams from HST/WFPC2 that reach below the horizontal branch for And I \& II; 2) searches for additional dwarf spheroidals that have revealed three new M31 companions. Consequently, this review will focus on these two areas of recent progress.

\section{WFPC2 Studies}

Although ground-based images of the M31 dSph companions can provide a large amount of useful information, such data are limited by image crowding to the brightest 2 or 3 magnitudes of the red giant branch. On the other hand, because of the Hubble Space Telescope's superior resolution, images obtained with the 
WFPC2 camera aboard HST can yield accurate photometry for stars at the level of the horizontal branch, or fainter, in these galaxies. In this section we outline some of the results obtained in our team's (other Co-Is are N. Caldwell and P. Seitzer) program to image the M31 dSph companions with WFPC2. The results for And I have been published in Da Costa et al. (1996) while those for And II are presented here for the first time. Our observations of And III are scheduled to be executed in early 1999 .

\subsection{Observations and Photometry}

Our program uses the $F 555 W$ (wide- $V$ ) and $F 450 W$ (wide- $B$ ) filters; the $F 450 W$ filter being preferred to the more common $F 814 W(\sim I)$ because it allows greater sensitivity to faint blue horizontal branch stars. For each dSph our procedure is to take a first series of deep exposures and then, a few $(\leq 5)$ days later, take a second series of exposures at the same orientation but with the field center displaced by $\sim 20$ PC pixels. This displacement of the field center allows us to readily separate faint stars from the instrumental effects present in WFPC2 images, while the lag between the observation sets allows us to detect variable stars, particularly RR Lyrae variables. The total exposure times for each $\mathrm{dSph}$ are of order $2.3 \mathrm{hrs}$ with the $F 555 \mathrm{~W}$ filter and $5.4 \mathrm{hrs}$ with $F 450 \mathrm{~W}$.

Even when centered on the centers of these dSph galaxies, the WFPC2 frames are sufficiently uncrowded (cf. Fig. 1 of Da Costa et al. 1996) that aperture photometry techniques can be successfully employed. We then use the zeropoints and transformation equations listed in Holtzman et al. (1995) to convert the WFPC2 photometry to the standard $V, B-V$ system, though we have a ground-based program underway to confirm the zeropoints of these data. The final color-magnitude diagram (CMD) for And I derived from our WFPC2 images is shown in Fig. 1, which is taken from Da Costa et al. (1996).

From the data presented in Fig. 1, and from the similar CMD for And II, we can draw a number of inferences. We will consider these in the following order: results that derive primarily from the morphology of the CMDs; results, such as dSph line-of-sight distances, that depend on the $F 555 \mathrm{~W} / \mathrm{V}$ zeropoint; and results, such as mean abundances and abundance dispersions, that depend on the $F 555 W / V$ zeropoint and the $F 450 W / B$ transformation and zeropoint.

\subsection{Horizontal Branch Morphologies}

It is immediately apparent from Fig. 1 that the morphology of the horizontal branch (HB) in And I is dominated by red HB stars. However, it is equally obvious that blue HB stars occur and, as Da Costa et al. (1996) have shown, $\mathrm{RR}$ Lyrae variables are also present. If we use a HB morphology index $i$ of the form $i=b /(b+r)$, where $b$ and $r$ are the numbers of blue and red HB stars, respectively (see $\mathrm{Da}$ Costa et al. 1996 for formal definition of these quantities), then $i=0.13 \pm 0.01$ for And I. As discussed below, the mean abundance for And $\mathrm{I}$ is $\langle[\mathrm{Fe} / \mathrm{H}]\rangle=-1.45 \pm 0.2 \mathrm{dex}$. At this abundance, the HB morphologies of Galactic globular clusters that follow the (HB morphology, $[\mathrm{Fe} / \mathrm{H}]$ ) relation defined by the majority of such clusters, have relatively many more blue HB stars and many fewer red HB stars than does And I. For example, the standard globular cluster M5, which has a similar abundance to And I, has $i \approx 0.75$. For this reason, we can say unequivocally that And I shows the second parameter 


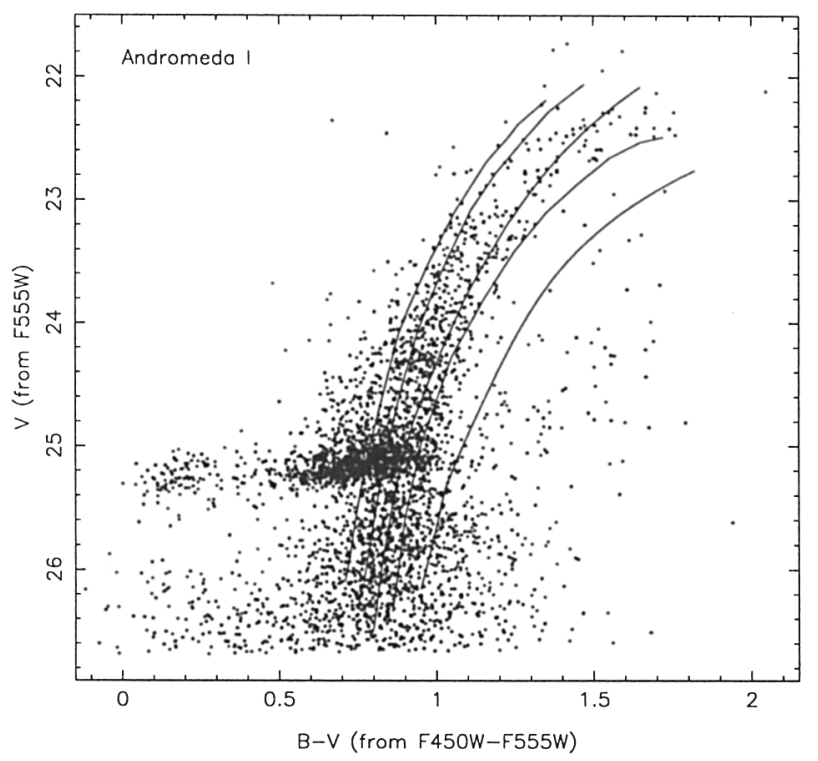

Figure 1. The $V,(B-V)$ color-magnitude diagram for the M31 dSph companion And I from Da Costa et al. (1996). Note the dominance of red horizontal branch (HB) stars though blue HB and RR Lyrae variables are also present. The giant branches of the standard Galactic globular clusters M68 ([Fe/H] $=-2.09)$, M55 (-1.82), NGC $6752(-1.54)$, NGC $362(-1.28)$ and 47 Tuc $(-0.71)$ are superposed assuming $(m-$ $M)_{V}=24.68$ and $\mathrm{E}(B-V)=0.04$ for And I, together with an additional 0.05 mag redward shift to improve the quality of the overall fit.

effect in the same way as do many of the Galactic dSph companions. Da Costa et al. (1996) go on to argue that in And I, the dominance of red HB stars results from a mean age for the bulk of the population that is younger than that of the Galactic globular clusters, though the presence of blue HB and RR Lyrae stars testifies to the presence of a minority older population. In other words, the And I CMD implies that star formation in this dSph has continued for an extended period. This interpretation is supported by the results of Mighell \& Rich (1996) for the Galactic dSph Leo II, which has similar HB morphology and mean abundance to And I. Mighell \& Rich (1996) used WFPC2 data that reaches below the main sequence turnoff to infer that the mean age of the stellar population in Leo II is $\sim 9$ Gyr, with the majority of the star formation having occurred in a $\sim 4$ Gyr interval about that epoch, though some older stars are also present.

Our WFPC2 CMD for And II is similar to that shown for And I in Fig. 1. In particular, once again the HB morphology is dominated by red stars though, as for And I, blue HB and candidate RR Lyrae variable stars are also present. Indeed, there are relatively more blue HB stars in the And II CMD than is the case for And I: the HB morphology index for And II is $i=0.18 \pm 0.02$ (cf. $i=0.13 \pm 0.01$ for And I). Given the somewhat higher mean abundance of And II $(<[\mathrm{Fe} / \mathrm{H}]>\approx-1.3$, see below $)$ and its large abundance dispersion, this 
$\mathrm{dSph}$ is less obviously a strong second parameter candidate in the way And I is. Nevertheless, it is likely that the second parameter effect is at work in And II. For example, the standard Galactic globular cluster M4, which has an abundance similar to the And II mean, has a relatively evenly populated HB with $i=0.45 \pm$ 0.05 , while NGC 362, which also has an abundance similar to the And II mean, has a dominant red HB morphology with $i=0.04 \pm 0.02$. This latter cluster is one of the 'classic' Galactic halo second parameter clusters and has an age $\sim 2$ Gyr younger than the majority of Galactic halo globular clusters. We conclude therefore, pending further analysis, that the And II CMD does show the second parameter effect and that the most likely cause is that the bulk of the stellar population in And II is somewhat younger than the age of most Galactic halo globular clusters.

Perhaps the most interesting result concerning HB morphology, however, is that in And I it varies with distance from the galaxy's center. Inside the core radius we find no radial variation in the HB morphology index, but outside the core radius the relative number of blue $\mathrm{HB}$ stars increases by approximately a factor of two. In other words, the blue HB stars in And I are more widely distributed than the red HB stars that make up the bulk of the population. The origin of this radial gradient is not easily identified but the most likely explanation is that at the epoch of its initial episode of star formation, the proto-And I was more spatially extended than it was when the bulk of the stars formed. Apparently similar HB morphology gradients are also present in two (Sculptor and Leo II) of the three Galactic dSphs where there is sufficient data to consider the question. We have not yet performed the equivalent analysis on our And II data.

\subsection{Distances}

For And I, the mean magnitude of the horizontal branch is $V=25.25 \pm 0.04$ mag. Adopting a reddening $\mathrm{E}(B-V)=0.04$ and the distance scale based on the horizontal branch models of Lee et al. (1990), in which $M_{V}(\mathrm{HB})=0.17[\mathrm{Fe} / \mathrm{H}]+$ $0.82 \mathrm{mag}^{2}$ the line-of-sight distance to And I is $810 \pm 30 \mathrm{kpc}$ for a mean abundance of $\langle[\mathrm{Fe} / \mathrm{H}]\rangle=-1.45 \pm 0.2 \mathrm{dex}$. This distance agrees well with that, 790 $\pm 60 \mathrm{kpc}$, derived by Mould \& Kristian (1990) from the $I$ magnitude of the And I red giant branch tip. The most directly comparable distance determinations for M31, based on either red giant branch tip stars or RR Lyraes in the M31 halo or horizontal branch stars in M31 globular clusters, are $760 \pm 45 \mathrm{kpc}$ or $850 \pm 20$ $\mathrm{kpc}$, both on the same distance scale (see the discussion in Da Costa et al. 1996). We can then conclude only that the relative M31/And I line-of-sight distance is $0 \pm 70 \mathrm{kpc}$. Given that And I lies $\sim 45 \mathrm{kpc}$ in projection from the center of M31, the true distance of And I from the center of M31 then lies between $\sim 45$ and $\sim 85 \mathrm{kpc}$. The lower limit is smaller than the galactocentric distances of any of the Galaxy's dSph companions with the exception of Sagittarius, while the upper limit is comparable to the galactocentric distances of the nearer Galactic dSphs such as Ursa Minor, Sculptor and Draco.

\footnotetext{
${ }^{2}$ This relation also underlies the $I$ magnitude of the red giant branch tip distance scale (cf. Da Costa \& Armandroff 1990, Lee et al. 1993).
} 
For And II, the mean magnitude of the horizontal branch is $V=24.90$ $\pm 0.04 \mathrm{mag}$ from which we can immediately infer that And II lies closer to us than And I. Indeed, adopting the same distance scale and reddening as above, and assuming a mean metal abundance of $\langle[\mathrm{Fe} / \mathrm{H}]\rangle \approx-1.3$ (see next section), we find that And II lies at a distance of $690 \pm 25 \mathrm{kpc}$, or $120 \pm 70 \mathrm{kpc}$ in front of M31 along the line-of-sight. This is consistent with the ground-based results of Konig et al. (1993) who found And II to be $0.4 \pm 0.2$ mag closer than M31. On the sky And II lies closer to M33 than M31. However, our results now unambiguously associate And II with M31 since M33 lies 150 kpc beyond M31 along the line of sight. When combined with And II's projected distance of $\sim 130$ $\mathrm{kpc}$ from the center of M31, these estimates indicate that the true distance of this dSph from the center of M31 lies between $\sim 150$ and $\sim 240 \mathrm{kpc}$. The lower limit corresponds approximately to the galactocentric distance of Fornax while the upper limit is similar to the galactocentric distances of the Leo systems, the most distant of the Galactic dSph companions. And II, however, may not be the most distant of the M31 dSph companions, since And VI (see below) lies at least $\sim 270 \mathrm{kpc}$ (the projected distance) from M31.

\subsection{Mean Metal Abundances and Abundance Dispersions}

With the distances established, the mean metal abundances of these dSphs can be determined via the comparison of the dSph CMDs with the giant branches of Galactic globular clusters whose $[\mathrm{Fe} / \mathrm{H}]$ values are well known. An example of this is shown in Fig. 1 where the giant branches of five standard globular clusters, whose abundances range from $[\mathrm{Fe} / \mathrm{H}]=-2.1$ to $[\mathrm{Fe} / \mathrm{H}]=-0.7$, are overlaid on the And I WFPC2 observations. Da Costa et al. (1996) discuss the mean abundance determination process in some detail and their conclusion is that the mean abundance of And $\mathrm{I}$ is $\langle[\mathrm{Fe} / \mathrm{H}]\rangle=-1.45 \pm 0.2$ dex. The uncertainty given includes uncertainty in the calibration relation, the statistical error in the observed giant branch mean color, and the effect of an adopted $\pm 0.03 \mathrm{mag}$ systematic uncertainty in the $B-V$ color zeropoint. This value, however, is in good accord with that, $\langle[\mathrm{Fe} / \mathrm{H}]\rangle=-1.4 \pm 0.2$, determined by Mould \& Kristian (1990) from the mean $(V-I)_{0}$ color of And I's upper red giant branch. Further, there does not appear to be any change in this mean abundance with distance from the center of And I.

Application of the same technique to the And II WFPC2 CMD yields a mean abundance of $\langle[\mathrm{Fe} / \mathrm{H}]\rangle \approx-1.3$, with an uncertainty comparable to that derived for And I. This mean abundance is somewhat more metal-rich than previous ground-based determinations. For example, Konig et al. (1993) give $<[\mathrm{Fe} / \mathrm{H}]>=-1.59(+0.44,-0.12)$ from their Gunn system $g, r$ CMD study while Armandroff (1994) lists $\langle[\mathrm{Fe} / \mathrm{H}]\rangle=-1.63 \pm 0.25$ from the mean giant branch color in a $(I, V-I)$ CMD. This relatively high mean abundance for And II derived from the WFPC2 data has an interesting consequence: at $\langle[\mathrm{Fe} / \mathrm{H}]\rangle$ $\approx-1.3$ and $M_{V} \approx-11.6$ (which is based on the new distance derived above), And II now deviates from the relation between mean abundance and absolute magnitude apparently followed by most $\mathrm{dSph}$ and $\mathrm{dE}$ galaxies (cf. Fig. 18 of Caldwell et al. 1998). For example, this mean abundance for And II exceeds that of the Fornax dSph, a system which is more than two magnitudes more luminous. 
The lack of image crowding on the WFPC2 images permits the photometric errors, unlike the case for ground-based images, to approach the photon statistics limit for all but the very brightest stars, where systematic effects seem to limit the precision to $\sim 0.015 \mathrm{mag}$ (see Da Costa et al. 1996). Consequently, investigating the intrinsic color width of the giant branch in these WFPC2 CMDs is relatively straightforward. The occurrence of internal ranges in abundance is one of the characteristics of the Galactic dSph galaxies and the M31 dSph companions prove to be no exceptions. For And I, the results of Da Costa et al. (1996) can be summarized as follows: on the upper part of the red giant branch, there is an intrinsic color spread that exceeds by a substantial factor the color spread expected on the basis of the photometric errors alone. Applying the same calibration as for the mean abundance, this color spread can be characterized in a number of ways. For example, $\sigma([\mathrm{Fe} / \mathrm{H}])=0.20 \mathrm{dex}$, the inter-quartile range is $0.30 \mathrm{dex}$, the central two-thirds of the sample abundance range is $0.45 \mathrm{dex}$, and the full range of the sample is $\sim 0.6 \mathrm{dex}$. These values are comparable to those seen in Galactic dSphs. For example, Suntzeff (1993) lists values of $\sigma([\mathrm{Fe} / \mathrm{H}])$ for five Galactic dSphs that range from $\sim 0.2$ to $\sim 0.3 \mathrm{dex}$.

On the other hand, Konig et al. (1993) have suggested that the internal abundance range in And II is unusually large, with $\sigma([\mathrm{Fe} / \mathrm{H}]) \approx 0.43 \mathrm{dex}$, though they admit that their photometric errors, which have to be subtracted in quadrature from their observed color dispersion, are large and not well constrained. Thus the validity of their result is questionable. However, our WFPC2 data appear also to indicate that the intrinsic abundance distribution in this $\mathrm{dSph}$ is quite broad, much broader than could be explained by the photometric errors alone. Further, because we have essentially two sets of data resulting from the two different pointings, our photometric errors are well determined (and small). Applying a similar analysis technique to that used by Da Costa et al. (1996) for And I, we find that $\sigma([\mathrm{Fe} / \mathrm{H}]) \approx 0.38$ dex for And II, while the inter-quartile range is $0.65 \mathrm{dex}$, the central two-thirds of the sample abundance range is 0.85 dex and the full range of the sample is $\sim 1.4$ dex. These numbers are significantly larger than those for And I. We thus have the interesting situation of two M31 companion dSphs, of similar luminosity and mean abundance, yet whose internal abundance ranges appear to be substantially different. This result reinforces the point that the internal abundance distributions of the Local Group dSphs apparently do not seem to readily correlate with luminosity in the way that the mean abundances do. Indeed the internal abundance distribution is a characteristic that has the potential to provide additional significant constraints on the star formation and enrichment histories in these galaxies. Further discussion of this point, however, is beyond the scope of this contribution.

\section{New Dwarf Companions to M31}

\subsection{Searches for Additional M31 Dwarf Spheroidals}

And I, II \& III were found by van den Bergh $(1972,1974)$ using the Palomar Schmidt and IIIaJ plates to survey 700 square degrees around M31. Subsequently, Kowal et al. (1978) reported three additional candidate Local Group systems (hence designation "LGS") found on IIIaJ Palomar Schmidt plates taken of an area that overlapped with, and extended to the south, the area surveyed 
by van den Bergh. Subsequent investigations have shown that only LGS 3 is actually a Local Group dwarf galaxy. It lies at a distance comparable to that of M31 and M33, but since it contains a modest amount of $\mathrm{H}$, I and a population of young stars (e.g. Aparicio et al. 1997 and the references therein), LGS 3 is not a dSph system and is thus not discussed here.

In recent years a number of new surveys for nearby dwarf galaxies have been undertaken. Irwin (1994) reported a survey for nearby dwarfs using automated star counts on sky survey plates covering over 20,000 square degrees of high-latitude sky. This survey revealed the Sextans dwarf spheroidal (Irwin et al. 1990). Whiting et al. (1997) visually examined survey plates of the entire southern sky, finding the Antlia dwarf. Neither of these surveys would be expected to reveal new M31 companions because, in the first instance, M31 is too distant for its companions to resolve into stars on sky survey plates and because, in the second instance, M31 is a northern object.

Because van den Bergh's $(1972,1974)$ survey yielded a total of three M31 dwarf spheroidals, when the Galaxy has nine, it has been recognized that additional M31 companions may be awaiting new surveys. Two other factors suggest that a new survey may be profitable: 1) the absolute magnitudes of And I, II \& III are significantly brighter than the faintest Galactic dwarf spheroidals (see Fig. 2 of Armandroff 1994); 2) the radial extent of van den Bergh's (1972, 1974) survey does not reach the galactocentric distances of the most distant Galactic dwarfs (see Fig. 6 of Armandroff 1994). Two surveys have recently been undertaken to find new M31 dwarfs.

The first survey, by Armandroff, Davies \& Jacoby (1998a; see also Armandroff et al. 1999a,b), uses digitized images from the Second Palomar Sky Survey (POSS-II; Reid et al. 1991; Lasker \& Postman 1993). The POSS-II has better resolution and depth than the POSS-I. Armandroff et al. (1998a) found that cleaning the POSS-II images of stars and bright galaxies, then applying a matched filter, easily reveals nearby low surface brightness dwarf galaxies. They optimized their filter and detection procedure for the known M31 dwarf spheroidals. To date, their detection procedure has been applied to 1550 square degrees around M31 (see Fig. 2). Once an object that resembles the known M31 dSphs on the processed and raw POSS-II images is found, small telescope CCD imaging is undertaken. This imaging eliminates most "contaminants" (e.g., distant galaxy clusters, distant low surface brightness spirals, Galactic cirrus clouds) and highlights true nearby dwarf galaxies by their incipient resolution into stars.

The second survey, by Karachentsev \& Karachentseva (1999), also uses the POSS-II and is part of their larger program to search the POSS-II films for nearby dwarf galaxies (see Karachentseva \& Karachentsev 1998). They visually searched a circular area of $22^{\circ}$ radius around M31, using morphological criteria to identify nearby low surface brightness dwarf candidates.

Armandroff et al. (1998a,b, 1999a,b) discovered two M31 dwarf spheroidal candidates: And V \& VI. Karachentsev \& Karachentseva $(1998,1999)$ found two strong candidate M31 dwarf galaxies: Pegasus Dwarf and Cas Dwarf. One candidate was found independently by both groups: And VI = Pegasus Dwarf. The Cas Dwarf is located in a region that lacked POSS-II digital data; therefore it was not found by Armandroff et al. (see Fig. 2). 


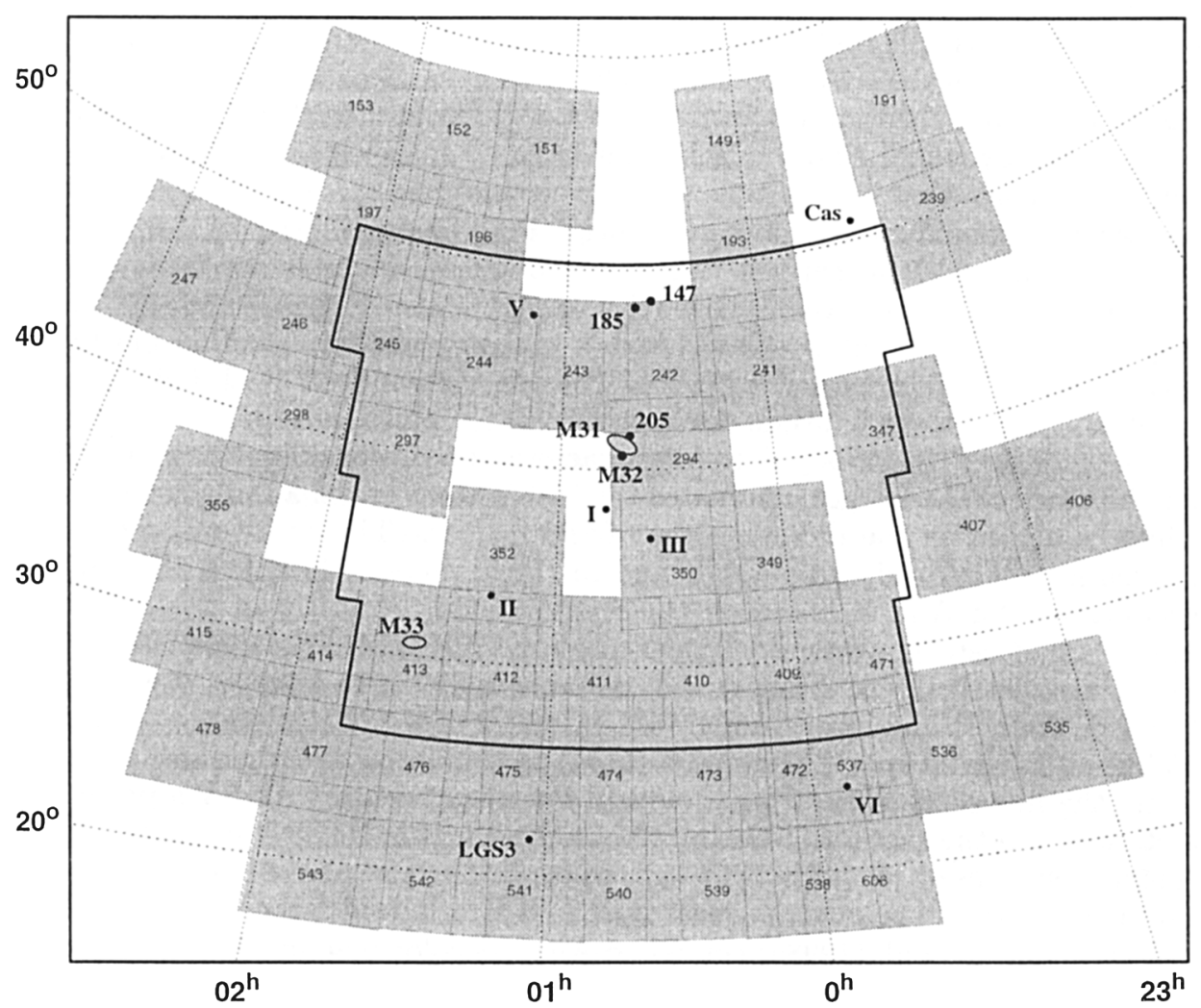

Figure 2. Map of the region of sky around M31 surveyed for low surface brightness dwarf galaxies by Armandroff et al. (1998a, 1999b), showing the POSS-II plates that have been searched. The area surveyed by van den Bergh $(1972,1974)$ is outlined in bold. M31, its known neighbors, and the new M31 dSphs And V, And VI and Cas are labeled.

\subsection{Properties of the Newly Discovered Dwarf Galaxies}

Three of the candidate M31 dwarf spheroidals found by Armandroff et al. and Karachentsev \& Karachentseva have been resolved into stars, indicating that they are indeed nearby dwarf galaxies. In this section, we summarize what is known about these three galaxies as of November 1998.

Armandroff et al. (1998a) presented follow-up observations of And V from the KPNO 4-m telescope and prime-focus CCD imager in the $V, R, I$ and $\mathrm{H} \alpha$ narrow-band filters. In the broad-band filters, And $\mathrm{V}$ resolves nicely into stars and exhibits a smooth stellar distribution, resembling the other M31 dwarf spheroidals. And V does not exhibit the features of classical dwarf irregulars, such as obvious regions of star formation or substantial asymmetries in its stellar distribution. In the And V continuum-subtracted $\mathrm{H} \alpha$ image, no diffuse $\mathrm{H} \alpha$ emission or $\mathrm{H}$ II regions are detected. The lack of $\mathrm{H} \alpha$ emission in And V reinforces the conclusion, based on And V's appearance on the broad-band images, 
that it is a dwarf spheroidal galaxy rather than a dwarf irregular. And V is not detected in any of the IRAS far-infrared bands either. Because far-infrared emission traces warm dust, and because some Local Group dwarf irregular galaxies are detected by IRAS, And V's lack of far-infrared emission serves as additional, weaker evidence that it is a dSph.

And V's apparent central surface brightness was measured by Armandroff et al. (1998a): $25.7 \mathrm{mag} \mathrm{arcsec}^{-2}$ in $V$. And $\mathrm{V}$ has a fainter apparent central surface brightness than And I, II \& III $\left(24.9,24.8\right.$, and $25.3 \mathrm{mag} \mathrm{arcsec}^{-2}$ in $V$, respectively; Caldwell et al. 1992). And V probably eluded detection until now due to its very dim apparent surface brightness. Armandroff et al. (1998a) also constructed a color-magnitude diagram for And V stars, in order to determine its distance and stellar populations characteristics. Color-magnitude diagrams for the parts of the images dominated by And V stars reveal a red giant branch, which is absent in the outer regions of the images. The tip of the red giant branch is well defined in the CMD and in the luminosity function. A distance was derived for And $\mathrm{V}$ based on the $I$ magnitude of the tip of the red giant branch (Da Costa \& Armandroff 1990, Lee et al. 1993). On the distance scale of Lee et al. (1990), the resulting And V distance is $810 \pm 45 \mathrm{kpc}$. As discussed in Sec. 2.3, the best and most comparable estimates of the M31 distance are $760 \pm$ $45 \mathrm{kpc}$ or $850 \pm 20 \mathrm{kpc}$. This implies that And V is located at the same distance along the line of sight as M31, to within the uncertainties. And V's projected distance from the center of M31 is $112 \mathrm{kpc}$; And I, II \& III have projected M31centric distances of 46,144 and $69 \mathrm{kpc}$, respectively. The above line-of-sight and projected distances strongly suggest that And V is indeed associated with M31.

Armandroff et al. (1998a) compared And V's color-magnitude diagram with fiducials representing the red giant branches of Galactic globular clusters that span a range of metal abundance (Da Costa \& Armandroff 1990). Based on the position of the And $\mathrm{V}$ giant branch relative to the fiducials, the mean metal abundance of And $\mathrm{V}$ is $\approx-1.5$, which is normal for a dSph (e.g., Fig. 9 of Armandroff et al. 1993). No bright blue stars are present in the And V CMD. Interpreting via isochrones, this lack of blue stars rules out any stars younger than $200 \mathrm{Myr}$ in And V and is further evidence that And V is a dwarf spheroidal and not a dwarf irregular. From the luminosities and numbers of upper asymptotic giant branch stars in a metal-poor stellar system, one can infer the age and strength of its intermediate age component (Renzini \& Buzzoni 1986). Using the And V field-subtracted luminosity function, there is no evidence for upper asymptotic giant branch stars that are more luminous than and redward of the red giant branch tip. Therefore, And V does not have a prominent intermediate age population; in this sense, it is similar to And I \& III.

And VI = Pegasus Dwarf has been resolved into stars and studied by two groups. Armandroff et al. (1998b) imaged And VI with the KPNO 4-m telescope prime-focus CCD on 1998 January 23 in $V$ for 300 seconds. And VI resolved nicely into stars in this short $V$ image, suggesting that it is indeed a nearby dwarf galaxy. On 1998 July 15, Armandroff et al. (1999b) obtained deeper And VI images with the KPNO 4-m telescope through the $R$ and $\mathrm{H} \alpha$ filters. The $R$ image of And VI exhibits a smooth stellar distribution and shows a resemblance to the other M31 dwarf spheroidals. And VI does not look lumpy or show obvious regions of star formation, suggesting that it is a dwarf spheroidal as opposed to 
a dwarf irregular. In the And VI continuum-subtracted $\mathrm{H} \alpha$ image, no diffuse $\mathrm{H} \alpha$ emission or $\mathrm{H}$ II regions are detected. The lack of $\mathrm{H} \alpha$ emission rules out current high-mass star formation in And VI and serves as further evidence that And VI is a dwarf spheroidal. Like And I, II, III \& V, And VI is not detected in any of the IRAS far-infrared bands. Armandroff et al. (1999b) are constructing a CMD for And VI based on images obtained with the WIYN 3.5-m telescope during excellent seeing conditions $(B, V \& I)$.

Grebel \& Guhathakurta (1999) have presented a CMD for And VI based on images from the Keck-II telescope in $V$ and $I$, which clearly shows the red giant branch. Based on the $I$ magnitude of the red giant branch tip, they determined a line-of-sight distance of $780 \pm 40 \mathrm{kpc}$, associating And VI with M31. From the position of the red giant branch relative to standard globular cluster fiducials, Grebel \& Guhathakurta found $<[\mathrm{Fe} / \mathrm{H}]\rangle=-1.2 \pm 0.3$. They also measured an intrinsic central surface brightness of $24.5 \mathrm{mag} \mathrm{arcsec}^{-2}$ in $V$.

Grebel \& Guhathakurta (1999) also obtained similar data for the Cas Dwarf from Keck-II. As for And VI, their CMD clearly shows the red giant branch, and from it they derived a distance and mean metallicity. Their line-of-sight distance of $710 \pm 35 \mathrm{kpc}$ places the Cas Dwarf in the extended M31 satellite system. The resulting metallicity is $\langle[\mathrm{Fe} / \mathrm{H}]\rangle=-1.3 \pm 0.3$. Grebel \& Guhathakurta also measured an extinction-corrected central surface brightness of $23.6 \mathrm{mag} \mathrm{arcsec}{ }^{-2}$ in $V$. Both And VI and the Cas Dwarf have apparent central surface brightnesses that are much brighter than And V, and somewhat brighter than And I, II \& III. Both And VI and the Cas Dwarf are outside of van den Bergh's (1972, 1974) survey area, which likely accounts for their anonymity until now.

Once accurate $M_{V}$ values are measured for And V, And VI and Cas, and once their $<[\mathrm{Fe} / \mathrm{H}]>$ values are established with definiteness, it will be important to re-evaluate whether the M31 and Galactic dSphs follow the same relations between absolute magnitude, central surface brightness, and mean metal abundance. The sample will be twice as large and the data of higher quality than the previous determinations (e.g., Fig. 7 of Armandroff 1994).

\subsection{Impact}

The discovery of the M31 dwarf spheroidals And V, And VI = Pegasus Dwarf, and Cas Dwarf doubles the number of known M31 dSphs. This changes the properties of M31's satellite system, as discussed below. The most obvious change is that M31 is not as poor in dwarf spheroidals as previously thought.

Karachentsev (1996) discussed the spatial distribution of the companions to M31. The discovery of And V, And VI and Cas changes the spatial distribution of the M31 satellites. Curiously, And I, II \& III are all located south of M31, while the three more luminous dwarf elliptical companions NGC 147, 185 \& 205 are all positioned north of M31. Also, Karachentsev (1996) noted that there are more M31 companions overall south of M31 than north of M31. The locations of And V and Cas north of M31 lessen both of these asymmetries (though And VI is south of M31; see Fig. 2). With projected radii from the center of M31 of 112, 271 and $224 \mathrm{kpc}$, respectively, And V, And VI and Cas increase the mean projected radius of the M31 dwarf spheroidals from $86 \mathrm{kpc}$ to $144 \mathrm{kpc}$.

The discovery of nearby dwarf galaxies like And V, And VI and Cas augments the faint end of the luminosity function of the Local Group. We do not 
yet have reliable $M_{V}$ values for And V, And VI or Cas, but they appear to be in the range $-12<M_{V} \leq-10$. This increases the number of galaxies in the Local Group in this $M_{V}$ range by $27 \%$. From a survey of nine clusters of galaxies, Trentham (1998) derived a composite luminosity function that is steeper at the faint end than that of the Local Group (see his Fig. 2). He attributed the difference to poor counting statistics and/or incompleteness among the Local Group sample. The discovery of And V, And VI and Cas reduces somewhat the discrepancy between the Local Group luminosity function and the extrapolation of Trentham's (1998) function.

\section{Future Directions}

Several opportunities for advancing our understanding of M31's dwarf spheroidal companions are apparent:

- The search for dSph companions to M31 is not yet complete. The ongoing searches based on the POSS-II may reveal additional companions. The Sloan Digital Sky Survey should allow a significantly deeper search for faint, low surface brightness M31 companions.

- It will be valuable to obtain HST/WFPC2 CMDs for all six M31 dwarf spheroidals. These CMDs will yield precise line-of-sight distances, hopefully facilitating a 3-dimensional map of the distribution of M31 satellites. Whether this distribution is random or exhibits "streams" will provide an interesting comparison with the Galactic dSph distribution. Also, having HB morphologies for all six known M31 dSphs will allow a more comprehensive evaluation of the second parameter effect in the halo of M31.

- No information is currently available about the H I content of And II, And V, And VI or Cas Dwarf via $21 \mathrm{~cm}$ observations. Either H I detections or strict upper limits would be valuable. The upper limits for And I \& III should be refined (see Thuan \& Martin 1979).

- Measurements of radial velocity are possible for red giants at the M31 distance with 8-10 meter telescopes. One can constrain the total mass of M31 via the velocity dispersion of its dwarf satellite system (as, for example, Zaritsky et al. (1989) did for the Galaxy). The impressive projected radii of And VI and Cas will result in mass limits to very large radii.

- Radial velocities will also allow mass-to-light ratios (M/L) to be derived for the M31 dSphs. Measurement of a high M/L for an M31 dSph would be a step toward universalizing the large velocity dispersions and consequent large $\mathrm{M} / \mathrm{L}$ values observed for Galactic dSphs. Also, the large galactocentric radii of And II, And VI and Cas should render tidal effects negligible.

Acknowledgements. We are grateful to our collaborators, Nelson Caldwell and Pat Seitzer on the And II WFPC2 CMD, and James Davies and George Jacoby on the M31 dSph survey, for allowing us to present results in advance of publication and for helpful discussions. 


\section{References}

Aparicio, A., Gallart, C., Bertelli, G. 1997, AJ, 114, 680

Armandroff, T.E. 1994, in: Dwarf Galaxies, (eds.) G. Meylan, P. Prugniel, ESO/OHP Workshop (Garching: ESO), p. 211

Armandroff, T.E., Da Costa, G.S., Caldwell, N., Seitzer, P. 1993, AJ, 106, 986

Armandroff, T.E., Davies, J.E., Jacoby, G.H. 1998a, AJ, 116, 2287

Armandroff, T.E., Davies, J.E., Jacoby, G.H. 1998b, in: Dwarf Tales, (eds.) E. Brinks, E.K. Grebel, Vol. 3, p. 2

Armandroff, T.E., Davies, J.E., Jacoby, G.H. 1999a, in: The Low Surface Brightness Universe, (eds) J.I. Davies, C. Impey, S. Phillipps (San Francisco: ASP), IAU Coll., 171, in press (astro-ph/9810299)

Armandroff, T.E., Jacoby, G.H., Davies, J.E. 1999b, AJ, to be submitted

Caldwell, N., Armandroff, T.E., Da Costa, G.S., Seitzer, P. 1998, AJ, 115, 535

Caldwell, N., Armandroff, T.E., Seitzer, P., Da Costa, G.S. 1992, AJ, 103, 840

Da Costa, G.S. 1998, in: Stellar Astrophysics for the Local Group, (eds.) A. Aparicio, A. Herrero, F. Sanchez (Cambridge: Cambridge Univ. Press) p. 351

Da Costa, G.S., Armandroff, T.E. 1990, AJ, 100, 162

Da Costa, G.S., Armandroff, T.E., Caldwell, N., Seitzer, P. 1996, AJ, 112, 2576

Grebel, E.K., Guhathakurta, P. 1999, ApJ, submitted

Holtzman, J., et al. 1995, PASP, 107, 1065

Irwin, M.J. 1994, in: Dwarf Galaxies, (eds.) G. Meylan, P. Prugniel, ESO/OHP Workshop (Garching: ESO) p. 27

Irwin, M.J., Bunclark, P.S., Bridgeland, M.T., McMahon, R.G. 1990, MNRAS, $244,16 \mathrm{P}$

Jones, J.H. 1993, AJ, 105, 933

Karachentsev, I. 1996, A\&A, 305, 33

Karachentsev, I., Karachentseva, V. 1998, in: Dwarf Tales, (eds.) E. Brinks, E.K. Grebel, Vol. 3, p. 1

Karachentsev, I.D., Karachentseva, V.E. 1999, A\&A, in press

Karachentseva, V.E., Karachentsev, I.D. 1998, A\&AS, 127, 409

Konig, C.H.B., Nemec, J.M., Mould, J.R., Fahlman, G.G. 1993, AJ, 106, 1819

Kowal, C., Lo, K.Y., Sargent, W.L.W. 1978, IAU Circular No. 3305

Lasker, B.M., Postman, M. 1993, in: Sky Surveys: Protostars to Protogalaxies, ASP Conf. Ser. 43, (ed). B.T. Soifer (San Francisco: ASP) p. 131

Lee, M.G., Freedman, W.L., Madore, B.F. 1993, ApJ, 417, 553

Lee, Y.-W., Demarque, P., Zinn, R. 1990, ApJ, 350, 155

Mighell, K.J., Rich, R.M. 1996, AJ, 111, 777

Mould, J., Kristian, J. 1990, ApJ, 354, 438

Reid, I.N., et al. 1991, PASP, 103, 661

Renzini, A., Buzzoni, A. 1986, in: Spectral Evolution of Galaxies, (eds.) C. Chiosi, A. Renzini (Dordrecht: Reidel) p. 195 
Suntzeff, N.B. 1993, in: The Globular Cluster - Galaxy Connection, (eds). G.H. Smith, J.P. Brodie, ASP Conf. Ser. 48, (San Francisco: ASP) p. 167

Thuan, T.X., Martin, G.E. 1979, ApJ, 232, L11

Trentham, N. 1998, MNRAS, 294, 193

van den Bergh, S. 1972, ApJ, 171, L31

van den Bergh, S. 1974, ApJ, 191, 271

van den Bergh, S. 1994, ApJ, 428, 617

Whiting, A.B., Irwin, M.J., Hau, G.K.T. 1997, AJ, 114, 996

Zaritsky, D., Olszewski, E.W., Schommer, R.A., Peterson, R.C., Aaronson, M. 1989, ApJ, 345, 759

\section{Discussion}

Lynden-Bell: May I advocate that you publish axis ratios and position angles for these systems from your star counts.

Armandroff: Thank you. We plan to do as you suggest.

Irwin: (1) What are the absolute magnitudes of And V and VI? (2) What are the detection limits of your survey? In particular, would the Galactic dSphs at $M_{V} \sim-8.5$ have been detected?

Armandroff: (1) We do not yet have surface brightness profiles for And V and VI, though we hope to soon. In our And V paper, we determined a dereddened central surface brightness of $25.2 \mathrm{~V} \mathrm{mag} \mathrm{arcsec}{ }^{-2}$. This suggests that And V has $M_{V} \sim-10.2$. (2) We have not yet carefully evaluated the completeness limits of our survey, although we hope to before the survey is completed. And V is a highly significant detection on the processed POSS-II images, so we expect that we should have the sensitivity to detect fainter dwarf spheroidals.

Zijlstra: Is M33 close enough to affect the orbits? Are the orbits stable?

Armandroff: I am not aware of detailed dynamical modelling of the M31 satellite system. M33 is located $\sim 250 \mathrm{kpc}$ from M31, and it is not clear whether it is bound or not. M33 and the outer M31 dwarfs have not completed that many orbits over the Hubble time, so the system is probably not fully virialized. Clearly, additional kinematic information and related modelling would be valuable.

Cannon: It's spectacular that two groups of people have between them found 3 new dwarf spheroidals around M31, doubling the known sample, in the last few months. What do you, or other experts here today, think the implications are for the Local Group as a whole? In other words, how many Tucana-like systems might be awaiting discovery?

Armandroff: We applied our enhancement and detection procedure to the sky around M31 because we expected additional low luminosity dwarfs there. Whiting/Irwin and Karachentsev/Karachentseva have been searching very large areas 
of sky using visual examination of the plates. Eventually, it may be worthwhile to apply our digital procedure to the POSS-II for large areas of sky away from M31.

Grebel: From follow-up on the Karachentseva and Karachentsev survey that we are doing in collaboration with them, there may be as many as 28 candidates for Local Group dwarf galaxies, similar to the one I showed earlier today. Groundbased follow-up on these will be done in the following months. We hope that a fraction of these object may turn out to the Local Group members. This work is done together with Karachentseva, Karachentsev, Seitzer, Guhathakurta, Sarajedini and Hodge. 